\title{
Development of compact accelerator neutron source
}

\author{
Alain Letourneau ${ }^{1, \text { a }}$, Anthony Marchix ${ }^{1}$, Ngoc-Hoang $\operatorname{Tran}^{1}$, Nicolas Chauvin ${ }^{1}$, Alain Menelle ${ }^{2}$, Frédéric Ott $^{2}$, \\ and Jérôme Schwindling ${ }^{1}$ \\ 1 Irfu, CEA, Université Paris-Saclay, 91191 Gif-sur-Yvette, France \\ 2 LLB, CEA, Université Paris-Saclay, 91191 Gif-sur-Yvette, France
}

\begin{abstract}
There is a worldwide growing interest for small-scale and reduced-cost neutron sources not based on nuclear fission. High-intensity proton or deuteron beams impinging on light materials could be used to produce such neutron sources with intensities or brightness comparable to nuclear reactor for dedicated experiments. To develop such technologies several key technological issues have to be addressed. Among them the neutron production and the maximization of the neutron extraction and transport to the instrument is a key parameter for the design of high-brightness sources adapted for the required application. This issue have to be addressed with validated and predictive Monte-Carlo simulations. In this paper we present preliminary results on the use of Geant4 in the context of Compact Accelerator based Neutron Source (CANS) developments.
\end{abstract}

\section{Introduction}

With the development of high-intensity accelerators it is now envisage to build compact high-intensity neutron sources for applications which are up to now only devoted to nuclear research reactors. The most symbolic project is the International Fusion Materials Irradiation Facility (IFMIF) using high-energy neutrons produced by the primary interaction between a deuteron beam and the lithium target for the qualification of materials which might be used in future nuclear fusion reactors. However, other applications leading to major industrial or societal challenges can be expected such as nuclear data measurements for nuclear industry, fundamental solid state physics studies with neutron scattering experiments, neutron radiography especially for industry materials qualification and also medical purposes like isotope production, neutron-capture therapy.

Compact Accelerator-driven Neutron Sources (CANS) are currently fast-growing leading to the founding in 2009 of the community UCANS (Union for Compact Accelerator-driven Neutron Sources) for promotion and stimulation in development of such sources especially for medical perspectives. They are attractive alternative solutions to nuclear research reactors especially in terms of public acceptance as well as for nuclear non-proliferation. Many facilities around the world have already chosen this option but it concerns accelerators with few $\mathrm{mA}$ of continuous current.

When dealing with high-intensity many challenges appear. First from the accelerator side, strong space charges can induce emittance growth, beam halo and beam losses that have to be controlled. The beam dynamics can be really challenging knowing that even very small losses can be harmful and can cause activation, quench of radio-frequency cavities and machine damages due to power deposition. Developing high-intensity neutron

a e-mail: aletourneau@cea.fr source really need expertise in high-intensity accelerator technology. Then from the target/moderator side, the heat removal is the main challenge for the target and the limiting parameter for the intensity. The optimization of the target/moderator assembly is a key ingredient to maximize the neutron transport onto the instrument. This optimization should be done taking into account the heat removal problem and the neutron features required by the users as for example the energy-pulse, the brightness and the shaping-time of the pulse. All these points could be addressed by using validated and predictive Monte-Carlo simulations.

In CEA, in France, we have started a work to promote such technology based on the existing $100 \mathrm{~mA}$ IPHI proton injector developed at Irfu/SACM. To provide an economical way of neutron production, low energy protoninduced reactions are considered. In our case less than $20 \mathrm{MeV}$ protons on thin beryllium-target are envisaged. As most of applications need moderated neutrons a lot of work has to be done to optimize the target-moderator assembly in order to maximize the neutron rate for the measurements.

In this paper we present the recent developments achieved on the simulation tools based on the Geant 4 Monte-Carlo code and the extrapolated performances for some applications showing good competitiveness with existing facilities.

\section{Compact neutron sources in the world}

Most of the new neutron sources (SNS, ISIS TS2, JPARC, ESS, CSNS) which have been or are being built in the world are based on spallation reactions with $\mathrm{GeV}$ - proton beams impinging on heavy metal targets $(\mathrm{Hg}, \mathrm{W}, \mathrm{Pb})$. As shown on Fig. 1 and Fig. 2, this reaction is the most efficient to produce neutrons but also the most costly. The shielding of the target is important due to very high energy

(C) The Authors, published by EDP Sciences. This is an Open Access article distributed under the terms of the Creative Commons Attribution License 4.0 (http://creativecommons.org/licenses/by/4.0/). 


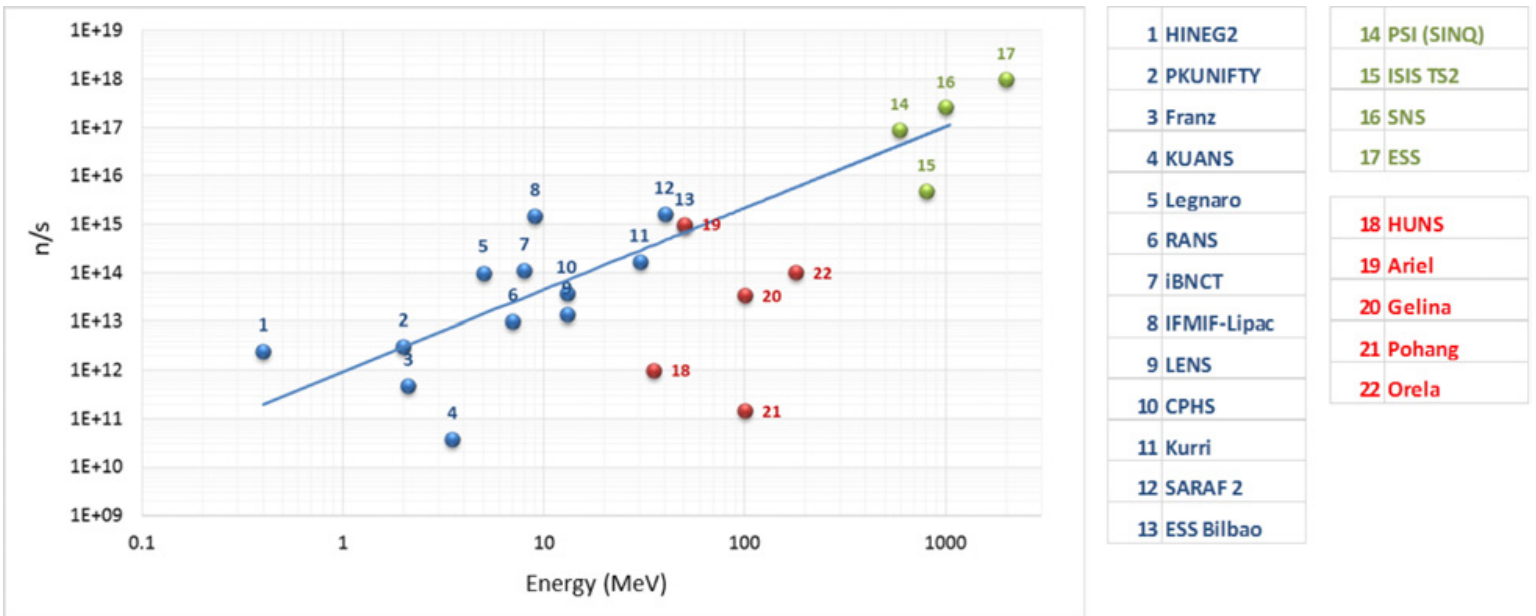

Figure 1. Existing and projected neutron sources classified as a function of their neutron brightness, incident beam energy and their technology: protons or deuterons on lithium or beryllium targets (from 1 to 13); protons on heavy metal targets (from 14 to 17) and electrons on heavy metal (from 18 to 22) [1]. CSNS does not figure on this plot but should appear between SNS and ESS. ESS Bilbao and Ariel have the same characteristics.

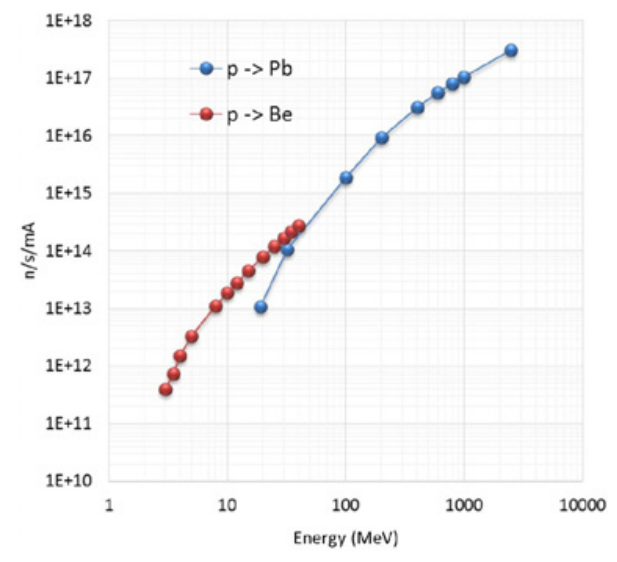

Figure 2. Calculated average neutron emission for protoninduced reactions on thick beryllium and lead targets [1]. Calculations were done using INCL4.6/ABLA07 models [2,4].

neutrons and $\gamma$-radiations. At ESS, the shielding of the target comprises about 6000 tons of steel.

Another approach is to use low energy reactions of protons or deuterons on lithium or beryllium targets. As shown on Fig. 2 such reactions produce several orders of magnitude less neutrons than spallation reactions, but partly compensated if using high-intensity beams. While a lithium target is producing low energy neutrons $(<1 \mathrm{MeV})$ which are easier to moderate, the low melting point of lithium would make it necessary to work with a liquid lithium target which is advantageous for the heat removal. The deuteron on beryllium reaction is about twice as more efficient as the proton on beryllium reaction at low-energy beam (less than $5 \mathrm{MeV}$ ) but a high intensity deuteron beam is more difficult to manage with respect to radioprotection issues. For these reasons most of the neutron sources in Fig. 1 use proton on beryllium target.

\section{The Geant4 simulation code}

The development of compact neutron sources requires accurate and validated simulation tools. On one hand, they are used to optimize the target-moderator-reflector assembly in terms of neutron fluency, time resolution and energy spectrum. On the other hand, they are used for dose rate calculations and shielding protections as radioprotection is a key issue for the construction of any new nuclear installation. The Monte Carlo simulation toolkit - Geant4 is a library for the simulation of the passage of particles through matter. It is used in large applications from high energy to very low energy of physics, including astrophysics and space science, medical physics and radiation protection [3]. For the present calculations, we used the version Geant4.10.02.

\subsection{Proton-induced reaction on beryllium}

The key ingredient to estimate the performances of a CANS installation is the neutron yield per incident proton. The final state probability in Geant 4 is a two-step process. First the probability of the process (electromagnetic, elastic or inelastic) is calculated and then the probability of the exit channel. Different models and/or nuclear data libraries can be used to compute these probabilities.

The Fig. 3 shows the calculated neutron yields for proton on thick beryllium target as a function of the incident proton energy for different options in Geant 4 and compared with existing data from Porges et al. [5], Scott [6] and Lone et al. [7]. In this comparison, we used the Barashenkov-Glauber inelastic cross section, as by default, and the inelastic cross sections from ENDF/B-VII.1 data library. The reaction channel probabilities were calculated using the three implemented intra-nuclear cascade models: the Bertini (Bert), the Binary Cascade (BinC) and the INCL ++ . The electromagnetic processes were calculated using the default Chips Elastic model. As seen on the figure calculations using the Barashenkov-Glauber cross section largely underestimate the neutron production whereas using the ENDF/B-VII.1 a better agreement is found with the experimental data even if at very low proton energy a factor two of discrepancy is observed for all the reaction channel models. A clear deviation of the binary cascade model is observed at higher proton energies resulting in a 


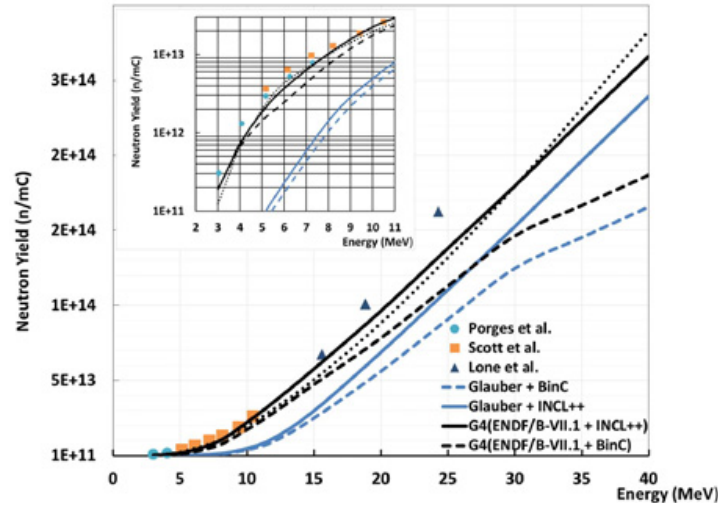

Figure 3. Neutron yields calculated (lines) for different options in Geant 4 and compared with existing experimental data (symbols) as a function of the incident proton energy on thick ${ }^{9}$ Be target. Experimental uncertainties are not indicated but should be of the order of $10-20 \%$.

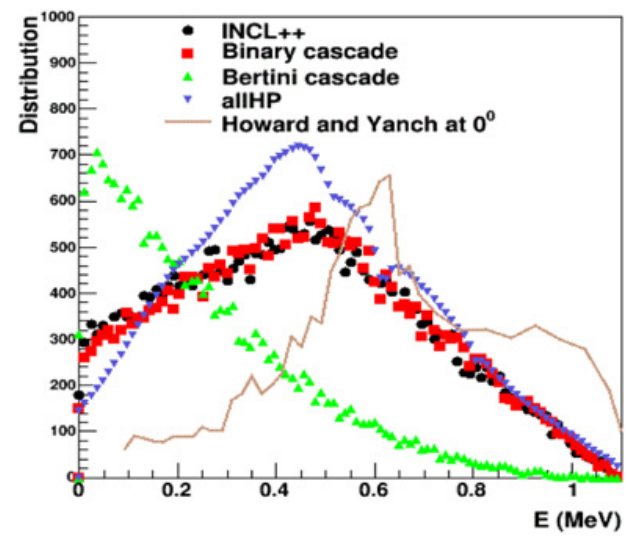

Figure 4. Neutron energy distributions calculated for the different intra-nuclear cascade models implemented in Geant 4 and in the case of using only nuclear data base (allHP) for $3 \mathrm{MeV}$ protons on thick ${ }^{9} \mathrm{Be}$ target. The data from Howard et al. [8] at $0^{\circ}$ are also plotted for comparison.

large underestimation of the neutron production with this model.

The angular and the energy distributions of emitted neutrons are also important to define the best strategy to moderate the neutrons and/or to extract them and to define the radiations shielding. Regarding the protonberyllium reaction differential cross sections are sparse below $20 \mathrm{MeV}$ incident protons with some discrepancies between the angular distributions for the oldest ones. Figure 4 and 5 show the energy and angular distributions calculated by Geant 4 for $3 \mathrm{MeV}$ incident protons on thick beryllium target using the intra-nuclear cascade models and the nuclear data libraries. The data from Howard and Yanch [8] are also plotted for comparison. All models, except Bertini cascade, have maximum energy around $450 \mathrm{keV}$. Bertini cascade model clearly underestimates the energy of emitted neutrons. All models agree on angular distributions mainly focused in the forward direction in agreement with Howard and Yanch data.

\subsection{Neutron transport and moderation}

The neutron transport was realized using the neutron-data library G4NDL4.5 based on the evaluated ENDF/B-VII

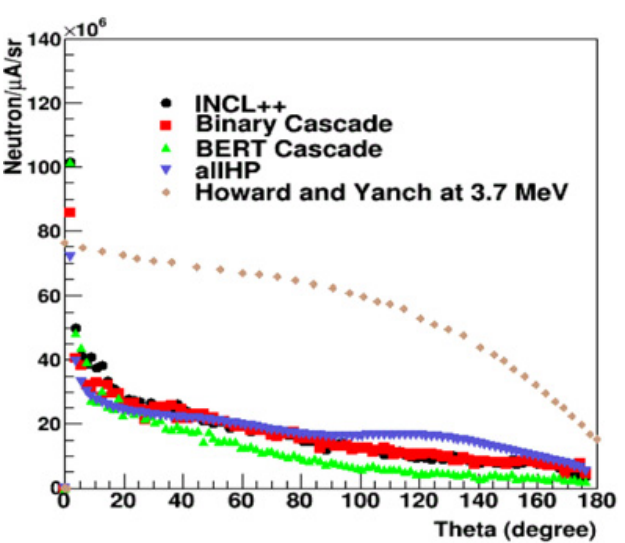

Figure 5. Idem than Fig. 4 for the angular neutron distributions.

nuclear data library. The thermal scattering library was also included below $4 \mathrm{eV}$ of neutron energy to take into account for molecular effects. In this work, we envisaged the neutron transport and moderation in polyethylene at ambient temperature.

\section{Experimental validations using the IPHI injector}

To test our Monte Carlo calculations and to evaluate the neutron performances we performed a test experiment using the high-intensity proton injector (IPHI). It consists in a proton source emitting $100 \mathrm{keV}$ protons (the SILHI source) and a Radio Frequency Quadrupole which accelerate protons up to energy of $3 \mathrm{MeV}$. The accelerator is designed to operate in continuous mode with proton currents up to $100 \mathrm{~mA}$ which correspond to a total power of $300 \mathrm{~kW}$. For the current experiment we have operated the accelerator at a very low power of about $10 \mathrm{~W}$ to avoid any target damage and for radioprotection purposes to limit the dose in the experimental room.

\subsection{Description of the experiment}

The proton beam was impinging on a thin beryllium target of thickness $0.5 \mathrm{~mm}$ screwed on an aluminium backing with titanium screws. The support was naturally air cooled to remove the heat deposit. The proton beam size was limited to $16 \mathrm{~mm}$ in diameter. The proton current incident on the target was continuously measured so as to have a precise value of the incident particle flux and to be able to precisely estimate the neutron production. An electron repelling electrode set at a potential of $200 \mathrm{~V}$ was set in front of the beryllium target to prevent any bias in the target current measurement.

The target was installed in a polyethylene (PE) block $\left(300 \times 300 \times 400 \mathrm{~mm}^{3}\right)$ to moderate neutrons to thermal energies. A $20 \mathrm{~mm}$ diameter extraction channel was drilled through the moderator to let the neutrons escape freely at $38^{\circ}$ from proton beam direction. The hole was drilled up to the depth where the thermal neutron density is expected to be the highest (see Fig. 6) at about $2.5 \mathrm{~cm}$ from the target.

The neutron production was measured inside the moderator using the activation of gold samples and at $8.4 \mathrm{~m}$ from the target using ${ }^{3} \mathrm{He}$ detectors at 5 bars. The 


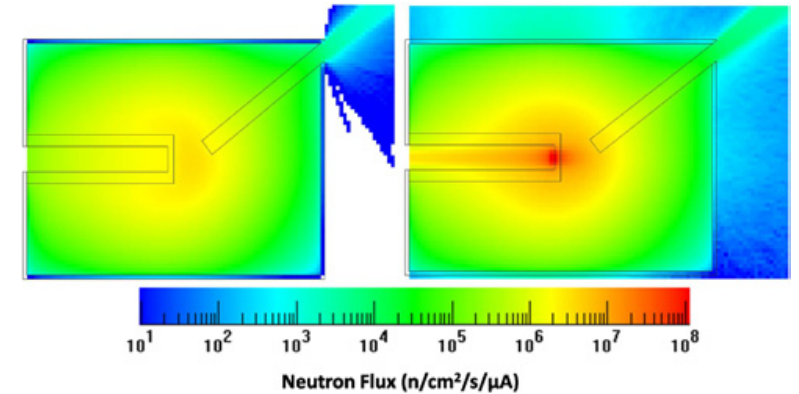

Figure 6. Simulated neutron density inside the polyethylene bloc for neutrons with energy less than $100 \mathrm{meV}$ (left) and all energies (right) given by the reference MCNPX 2.6 code [9].

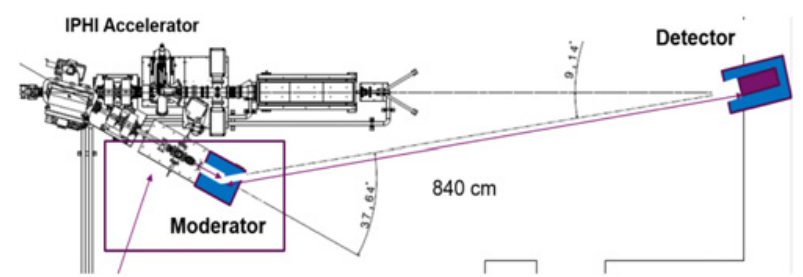

Figure 7. Scheme of the experimental set-up using the IPHI injector.

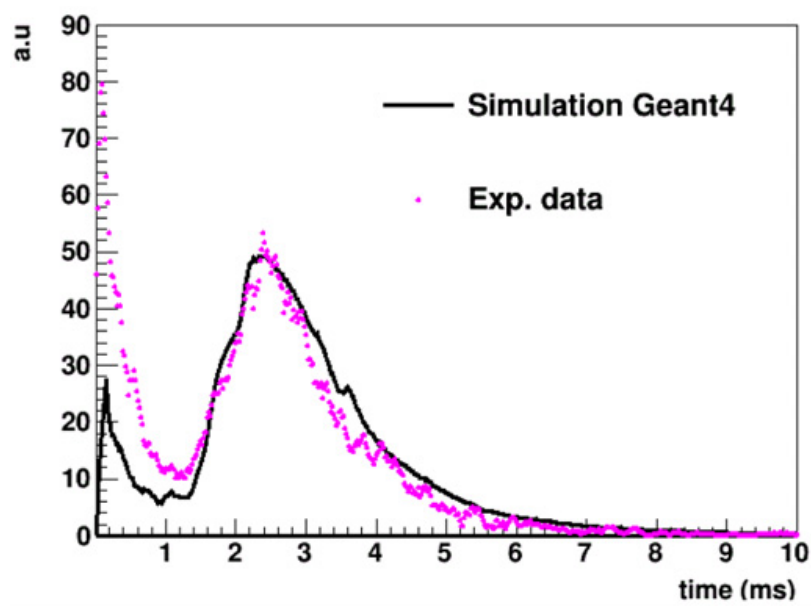

Figure 8. Neutron time of flight distribution measured (symbols) and compared with the simulation (not corrected from the $100 \mu \mathrm{s}$ pulse width).

RFQ signal of the accelerator was taken for the start of the measurement. The accelerator was operated in pulsed mode with pulses of length $100 \mu$ s and with a repetition rate of $1 \mathrm{~Hz}$. The rather sharp pulses allowed precise timeof-flight measurements with the ${ }^{3} \mathrm{He}$ counter.

\subsection{Results and perspectives}

The preliminary analysis of gold samples shows a good agreement with the simulation validating the calculated neutron flux inside the moderator. The preliminary result on time of flight measurements shows a quite good agreement on the shape for thermal neutrons (above $1 \mathrm{~ms}$ ) whereas the higher energy region (below $1 \mathrm{~ms}$ ) seems to be underestimated (see Fig. 8). This point should be investigated further and comparisons with MNCPX calculations done. These results are very encouraging to assess the abilities of Geant 4 to be used for the design of low-energy neutron sources.

From these results we can extrapolate the neutron brilliance for an installation based on $100 \mathrm{~mA}$ and $20 \mathrm{MeV}$ protons on beryllium target. A gain in the neutron yield of a factor 200 can be achieved by increasing the proton energy from $3 \mathrm{MeV}$ to $20 \mathrm{MeV}$. The brilliance at the surface of the moderator would be of about $4.10^{11} \mathrm{n} / \mathrm{cm}^{2} / \mathrm{s}$ that is 3 orders of magnitude less than the brilliance at the entrance of the guide systems at the Orphée reactor $\left(1.510^{14} \mathrm{n} / \mathrm{cm}^{2} / \mathrm{s}\right)$ for example. With optimized moderator geometry and neutron extraction we could expect to gain in brilliance on the instrument. However, such beam will deposit about $2 \mathrm{MW}$ of power on the target that will be difficult to dissipate. The source should probably be operated in pulsed mode with a typical duty cycle of the order of $2-4 \%$. This would correspond to a more manageable power load on the target of the order of $40-80 \mathrm{~kW}$. Further investigation should be performed to improve the heat dissipation.

\section{References}

[1] The SONATE project, internal note, CEA (2016)

[2] A. Boudard et al., Phys. Rev. C. 87, 014606 (2013)

[3] J. Allison et al., Nucl. Inst. Meth. A 835, 186 (2016)

[4] A.R. Junghans et al., Nuc. Phys. A 629, 635 (1998)

[5] K. Porges et al., Progr. Rep., ANL7910, 361 (1971)

[6] M.C. Scott, J. Nucl. Energy 25, 405 (1971)

[7] M.A. Lone et al., Nucl. Inst. Meth. 143, 331 (1977)

[8] W.B. Howard and J.C Yanch, Nuc. Sci. and Eng. 138, 145 (2001)

[9] D.B. Pelowitz et al., "MCNPX User's Manual, V2.6.0”, LANL Report LA-CP-07-1473 (2008) 